\title{
Envelhecimento acelerado em sementes de sorgo sacarino Sorghum bicolor (L.) Moench
}

\section{Accelerated aging in sorghum sacarino seeds Sorghum bicolor (L.) Moench}

\author{
Cristiane Deuner $^{1 *}$, Andréia da Silva Almeida ${ }^{2}$, Matheus Luiz Testa ${ }^{3}$, Anna dos Santos Suñe ${ }^{4}$, Lilian Madruga de Tunes ${ }^{5}$
}

Resumo: O sorgo sacarino é cultivado a partir de sementes, necessitando técnicas adequadas de produção, colheita e secagem das mesmas para garantir sua disponibilidade e qualidade. Objetivou-se avaliar a qualidade inicial estudando uma metodologia mais adequada do teste de envelhecimento acelerado para a avaliação do potencial fisiológico de sementes de sorgo sacarino. A pesquisa foi conduzida no Laboratório Didático de Análise de Sementes do Departamento de Fitotecnia, Universidade Federal de Pelotas, em Pelotas, Rio Grande do Sul. Utilizou-se delineamento experimental inteiramente casualizado com 4 repetições. Foram utilizados quatro lotes de sementes de sorgo sacarino. Inicialmente, foi avaliada a qualidade inicial das sementes através dos seguintes testes: teor de água, germinação, primeira contagem de germinação e emergência a campo. Após, os lotes foram submetidos ao teste de envelhecimento tradicional e com uso se solução salina saturada (NaCl), nos períodos de $12,24,48,72,96$ e $120 \mathrm{~h}$ à $41^{\circ} \mathrm{C}$. A exposição das sementes por $24 \mathrm{~h}$ a $41^{\circ} \mathrm{C}$ no envelhecimento acelerado com uso de solução saturada de $\mathrm{NaCl}$ e por $48 \mathrm{~h}$ a $41^{\circ} \mathrm{C}$ no envelhecimento acelerado tradicional são adequados para diferenciar lotes de sementes de sorgo sacarino.

Palavras-chave: Potencial fisiológico; Germinação; Vigor.

\begin{abstract}
Saccharin sorghum is cultivated from seeds, requiring adequate production, harvesting and drying techniques to ensure the availability of quality seed and, therefore. The objective was to evaluate the initial quality by studying a more adequate methodology of the accelerated aging test for the evaluation of the physiological potential of sorghum seeds. The research was conducted in the Laboratory of Seed Analysis of the Department of Plant Science, Federal University of Pelotas, in Pelotas, Rio Grande do Sul. A completely randomized experimental design with 4 replicates was used. Four lots of sweet sorghum seeds were used. First we assessed the quality of the seeds by the following tests: water content, germination, first count and emergency field. Following evaluation of initial quality, lots were submitted to the traditional aging test and use saturated saline, in periods of 12, 24, 48, 72, 96 and 120 hours at $41{ }^{\circ} \mathrm{C}$. Exposure of seeds for 24 hours at $41{ }^{\circ} \mathrm{C}$ in accelerated aging with saturated $\mathrm{NaCl}$ and use for $48 \mathrm{~h}$ at $41^{\circ} \mathrm{C}$ in traditional accelerated aging are suitable to differentiate batches of sweet sorghum seeds.
\end{abstract}

Key words: Physiological potential; Germination; Vigor.

\footnotetext{
*Autor para correspondência

Recebido para publicação em 08/07/2016; aprovado em 20/05/2017

${ }^{1}$ Dra. Prof. ${ }^{a}$ no IFSul - Câmpus CaVG. Pelotas, RS. E-mail: cdeuner@yahoo.com.br

${ }^{2}$ Pós doutoranda - PNPD institucional do Programa de Pós-Graduação em Ciência e Tecnologia de Sementes - UFPel. E-mail: andreiasalmeida@yahoo.com.br

${ }^{3}$ Msc. em Ciência e Tecnologia de Sementes - UFPel. E-mail: matheus.testa@outlook.com

${ }^{4}$ Doutoranda do Programa de Pós-Graduação em Ciência e Tecnologia de Sementes - UFPel. E-mail: annassune@gmail.com

${ }^{5}$ Dra. Prof. a do Programa de Pós-Graduação em Ciência e Tecnologia de Sementes - UFPel. E-mail: lilianmtunes@yahoo.com.br
} 


\section{INTRODUÇÃO}

O sorgo sacarino (Sorghum bicolor (L.) Moench) é nativo da Âfrica, possui alto potencial forrageiro e, dentre outras vantagens, pode-se citar rapidez no ciclo, fonte para alimentação humana, animal ou para a produção de biocombustível e utilização do bagaço como fonte de energia para industrialização (PARRELLA, 2012).

Por adaptar-se a vários ambientes, principalmente os que possuem deficiência hídrica e alta temperatura, esse sorgo se torna uma excelente alternativa para as diversas regiões brasileiras (SANTOS et al., 2005), além de ser uma opção para a produção de etanol. De acordo com Moreira et al. (2010) a previsão para o esgotamento das fontes de petróleo para o futuro próximo e a necessidade de proteção ambiental tem tornado cada vez mais necessária a busca por combustíveis alternativos aos derivados de petróleo, como por exemplo, o etanol. O sorgo sacarino, juntamente com a canade-açúcar e a beterraba açucareira estão entre as culturas de grande potencial energético (SOUZA et al., 2005).

O sorgo sacarino se assemelha à cana-de-açúcar, uma vez que o armazenamento do açúcar ocorre no colmo, além de fornecer bagaço para a indústria. Entretanto, ele difere de maneira acentuada da cana-de-açúcar pelo fato de ser cultivado a partir de sementes. Nesse contexto, há a necessidade de uso técnicas adequadas de produção, colheita e secagem das sementes para garantir a sustentabilidade do sistema (PARRELLA; PARRELLA, 2011). Segundo Durães (2011), a disponibilidade de sementes de qualidade é imprescindível para ganhos de produtividade e rendimento, e adequadas técnicas de plantio e de manejo da cultura são limitantes para a melhor performance agronômica e produtiva. Ainda, de acordo com Parrella e Parrella (2011), com a perspectiva de crescimento da cultura do sorgo sacarino como matéria prima alternativa na produção de etanol, aumentará significantemente a demanda por sementes de alta qualidade.

Para avaliar a qualidade e identificar diferenças no desempenho de lotes de sementes, diversos testes de vigor são utilizados, com o objetivo de que os resultados expressem o potencial de desempenho do lote de sementes sob condições de campo (DUTRA; VIEIRA, 2004), visando assim, complementar informações fornecidas pelo teste de germinação (MARCOS FILHO; NOVEMBRE, 2009) que, embora muito útil, não informa sobre o vigor e emergência em campo (MENEZES, 2001).

Dentre estes testes está o envelhecimento acelerado, no qual as sementes são submetidas a níveis adversos de temperatura e umidade relativa. O teste é utilizado para a avaliação do potencial fisiológico de sementes de várias espécies, tais como trigo, azevém, soja, milho, arroz, (DUTRA; VIEIRA, 2004; LOPES et al., 2009; PEDROSO et al., 2010; VENANCIO et al., 2012; TUNES et al., 2012). Porém, algumas sementes embebem e deterioram-se mais rapidamente, dificultando a obtenção de resultados confiáveis (TORRES; MARCOS FILHO, 2001) e devido a isso, a condução do teste tem sido realizada também com a substituição da água por soluções saturadas de sais, permitindo a obtenção de maior eficiência do teste na classificação dos lotes. Tunes et al. (2011) concluíram que o uso de solução salina saturada ou não saturada de $\mathrm{NaCl}$ contribui para o aprimoramento da metodologia do teste de envelhecimento acelerado na avaliação do vigor de sementes de azevém.

Segundo Vazquez et al. (2011), para sementes de sorgo, não há consenso quanto à temperatura e o período de exposição das sementes para o teste de envelhecimento acelerado. Souza e Marcos Filho (1975) avaliaram a exposição de sementes de sorgo ao envelhecimento acelerado pelos períodos de 120 e 168 horas a $42{ }^{\circ} \mathrm{C}$ e observaram resultados muito drásticos, sugerindo mais estudos. Em contrapartida, Ibrahim et al. (1993) concluíram que a combinação de $43{ }^{\circ} \mathrm{C}$ por $72 \mathrm{~h}$ é eficiente para a separação dos lotes de sementes de sorgo em diferentes níveis de vigor. Outros trabalhos com sorgo indicaram a combinação $41{ }^{\circ} \mathrm{C}$ por $96 \mathrm{~h}$ de exposição das sementes como sendo a mais promissora para a separação de lotes de sementes (MARCOS FILHO, 1994; MIRANDA et al., 2001; VAZQUEZ et al., 2011).

Nesse sentido, objetivou-se avaliar a qualidade inicial estudando uma metodologia mais adequada do teste de envelhecimento acelerado para a avaliação do potencial fisiológico de sementes de quatro lotes de sorgo sacarino verificando sua relação com a emergência a campo.

\section{MATERIAL E MÉTODOS}

O estudo foi realizado no Laboratório Didático de Análise de Sementes do Departamento de Fitotecnia, Faculdade de Agronomia "Eliseu Maciel", Universidade Federal de Pelotas, em Pelotas (RS), com quatro lotes de sementes de sorgo sacarino, denominados Lote 1, 2, 3 e 4.

Inicialmente foi avaliada a qualidade inicial das sementes através dos seguintes testes: Teor de água: determinado de acordo com as Regras para Análise de Sementes (RAS), através do método da estufa $105 \pm 3{ }^{\circ} \mathrm{C}$, utilizando-se duas subamostras de sementes para cada lote (BRASIL, 2009); Germinação: conduzido com quatro repetições de 50 sementes para cada repetição, distribuídas em rolos de papel germitest umedecido com água destilada na proporção de 2,5 vezes o seu peso seco. Os rolos foram mantidos em germinador a $25^{\circ} \mathrm{C}$, e as contagens realizadas aos quatro e 10 dias após a semeadura, conforme as RAS (BRASIL, 2009), computando-se a percentagem de plântulas normais; Primeira contagem da germinação: realizado simultaneamente ao teste de germinação, sendo as contagens realizadas no quarto dia após instalação do teste, computando-se a percentagem de plântulas normais (BRASIL, 2009); Emergência em campo: determinada com quatro repetições de 50 sementes por lote, semeadas a $2 \mathrm{~cm}$ de profundidade. A avaliação foi efetuada aos 10 dias após a semeadura, determinando a porcentagem de plântulas normais emergidas segundo Nakagawa (1999).

Após a avaliação da qualidade inicial, os lotes foram submetidos ao teste de envelhecimento tradicional e modificado, conforme descrito a seguir: Envelhecimento acelerado tradicional: utilizou-se caixas plásticas tipo gerbox, contendo $40 \mathrm{~mL}$ de água destilada e uma tela de alumínio suspensa, onde as sementes foram distribuídas formando uma camada uniforme; e Envelhecimento acelerado com uso de solução salina saturada: conduzido com metodologia similar ao envelhecimento acelerado tradicional, porém, adicionandose ao fundo da caixa plástica, $40 \mathrm{~mL}$ de solução de $\mathrm{NaCl}(40 \mathrm{~g}$ de $\mathrm{NaCl}$ em $100 \mathrm{~mL}$ de água), estabelecendo ambiente com umidade relativa de 76\% (JIANHUA; McDONALD, 1996). 
As caixas, de ambos os testes, foram mantidas a $41{ }^{\circ} \mathrm{C} \mathrm{em}$ câmara tipo BOD, durante 12, 24, 48, 72, 96 e 120 horas, sendo então, quatro repetições de 50 sementes por lote submetidas ao teste de germinação, conforme descrito anteriormente, com avaliação realizada no quarto dia após a instalação do teste.

Também foi determinado o teor de água das sementes após cada período de envelhecimento, para verificar a uniformidade das condições do teste, conforme Marcos Filho (1999).

O delineamento experimental foi inteiramente casualizado com 4 repetições, sendo as médias comparadas pelo teste de Tukey ao nível de 5\% de probabilidade e os coeficientes de correlação através do método de Pearson, a 1 e a 5\% de probabilidade, através do Programa estatístico WinStat 1.0 (MACHADO; CONCEIÇÃO, 2003).

\section{RESULTADOS E DISCUSSÃO}

Analisando os resultados da qualidade inicial dos lotes de sorgo sacarino, observou-se que não houve diferença significativa entre os lotes para os testes de germinação e primeira contagem de germinação, porém, houve diferença para a emergência a campo, sendo que o lote 2 apresentou-se superior e o lote 4 inferior aos demais (Tabela 1). De acordo com Tunes et al. (2011) a discordância entre os resultados obtidos nos testes de qualidade inicial sugere a necessidade de realização de outros testes antes de classificar os lotes quanto ao potencial fisiológico, pois cada teste tem um princípio diferente e fornece informações complementares para o destino final de cada lote de sementes.

Tabela 1. Qualidade inicial de quatro lotes de sementes de sorgo sacarino, avaliada pelos testes de germinação (GER), primeira contagem da germinação (PCG) e emergência em campo (EC).

\begin{tabular}{cccc}
\hline Lotes & GER & PCG & EC \\
\hline 1 & $91 \mathrm{~A}^{*}$ & $81 \mathrm{~A}$ & $82 \mathrm{BC}$ \\
2 & $94 \mathrm{~A}$ & $82 \mathrm{~A}$ & $95 \mathrm{~A}$ \\
3 & $89 \mathrm{~A}$ & $83 \mathrm{~A}$ & $85 \mathrm{~B}$ \\
4 & $90 \mathrm{~A}$ & $79 \mathrm{~A}$ & $76 \mathrm{C}$ \\
\hline $\mathrm{CV}(\%)$ & 2,7 & 3,6 & 4,9 \\
\hline
\end{tabular}

*Médias seguidas pela mesma letra na coluna não diferem entre si pelo teste de Tukey a $5 \%$ de probabilidade.

Os resultados referentes ao teor de água inicial e após envelhecimento acelerado com e sem uso de solução de $\mathrm{NaCl}$ não foram analisados estatisticamente e estão apresentados na Tabela 2. Inicialmente, os teores de água foram baixos e semelhantes entre os lotes, variando de 11,1 a $12,3 \%$, o que permite uma maior confiabilidade dos resultados obtidos (Tunes et al., 2011). Coimbra et al. (2009) salientam que o teor de água inicial das sementes é um fator primordial para a padronização dos testes de avaliação de qualidade.

A medida que aumentou o período de exposição ao envelhecimento houve também um aumento nos teores de água em todos os lotes e em ambos os métodos. Porém, no envelhecimento acelerado tradicional observa-se que ocorreu um aumento brusco no teor de água no período de 24 para 48h de exposição, enquanto que no envelhecimento acelerado com solução saturada de sal, o incremento foi gradual em todos os períodos, permitindo resultados mais uniformes, exceto para o lote 1 que apresentou incremento brusco no período de 72 para $96 \mathrm{~h}$ de exposição. Resultados semelhantes foram observados por Lemes et al. (2015) em sementes de grama-bermuda. Segundo os autores, ocorreram maiores acréscimos nos teores de água no teste de envelhecimento tradicional, com maior velocidade de captação de água pelas sementes, quando comparado às soluções salinas (saturada e não saturada). Segundo Jain et al. (2006), o aumento no grau de umidade das sementes pode ser explicado pela desorganização das membranas celulares durante o teste de envelhecimento das sementes.

Analisando os resultados da germinação das sementes após a submissão ao envelhecimento acelerado tradicional e com $\mathrm{NaCl}$ (Tabela 3), observou-se que não houve diferença quanto ao vigor dos lotes após $12 \mathrm{~h}$ de exposição, em ambos os métodos, e após 24h no envelhecimento acelerado tradicional. Verificou-se também, que após 24 h de exposição ao envelhecimento acelerado com solução salina saturada foi possível classificar os lotes em 3 níveis de vigor, sendo que o 2 e o 3 mantiveram-se com maior vigor até o período de $96 \mathrm{~h}$ de exposição. No envelhecimento tradicional foi possível diferenciar os lotes em quatro níveis de vigor, após $48 \mathrm{~h}$ de exposição, sendo o 2 o que apresentou maior porcentagem de germinação e o lote 4 a menor.

Os testes de $24 \mathrm{~h}$ de exposição a $41{ }^{\circ} \mathrm{C}$ no EASSS e de $48 \mathrm{~h}$ no EAT se mostraram adequados para avaliar o vigor de sementes de sorgo sacarino. Considerando que o almejado em um teste de vigor é que além de informações confiáveis e que aponte a diferença entre os lotes, ele seja rápido.

Miranda et al. (2001) avaliando o envelhecimento acelerado em sementes de sorgo observaram que o teste foi eficiente para identificar diferenças entre lotes de alto e baixo potencial fisiológico, sendo as combinações mais adequadas a de $41{ }^{\circ} \mathrm{C}$ por 96 horas e $43{ }^{\circ} \mathrm{C}$ por 72 horas. Vazquez et al. (2011) também verificaram que o teste de envelhecimento acelerado, a $41{ }^{\circ} \mathrm{C}$ por 96 horas de exposição, foi capaz de promover uma adequada estratificação de lotes de sementes de sorgo em diferentes níveis de vigor. Avaliando o potencial fisiológico de sementes de nabo forrageiro, Nery et al. (2009), concluíram que o teste de envelhecimento acelerado tradicional, a $41^{\circ} \mathrm{C}$, durante 96 horas e com solução saturada, a $41{ }^{\circ} \mathrm{C}$ por $72 \mathrm{~h}$ foi eficiente para distinguir os lotes.

Tunes et al. (2012) avaliando o envelhecimento acelerado como teste de vigor para sementes de arroz observaram que a opção $24 \mathrm{~h}$ com solução saturada de $\mathrm{NaCl}$ ou $48 \mathrm{~h}$, com solução não saturada, promoveu uma melhor estratificação dos lotes de sementes. Em sementes de trigo, Pedroso et al. (2010), concluíram que o teste de envelhecimento acelerado com solução saturada de $\mathrm{NaCl}$, no período de $96 \mathrm{~h}$ a $42{ }^{\circ} \mathrm{C}$, é o mais adequado para avaliar o potencial fisiológico das sementes, em alto, médio e baixo vigor.

Na Tabela 4 estão apresentados os coeficientes de correlação de Pearson. Os resultados mostraram que houve correlação positiva entre o teste de germinação com o EASSS no período de $12 \mathrm{~h}$ de exposição e da emergência a campo com todos os períodos de ambos os métodos, exceto para $12 \mathrm{e}$ $24 \mathrm{~h}$ no EAT, inferindo que o teste de envelhecimento acelerado é indicado para avaliar o potencial fisiológico de sementes de sorgo sacarino.

Guiscem et al. (2001) também observaram correlação significativa e positiva entre o teste de envelhecimento acelerado e a emergência das plântulas no campo em sementes de milho doce BR 400. Por outro lado, Braz e 
Rossetto (2009) verificaram, que o teste de envelhecimento acelerado tradicional e com uso de solução salina não apresentou correlação significativa com a emergência em sementes de girassol. Bertolin et al. (2011) avaliando sementes de feijão também não encontraram correlação significativa entre o envelhecimento acelerado e a emergência de plantas.

Tabela 2. Teores de água (\%) inicial e após os períodos de envelhecimento acelerado tradicional (EAT) e com solução salina saturada (EASSS) de quatro lotes de sementes de sorgo sacarino

\begin{tabular}{|c|c|c|c|c|c|c|c|c|c|c|c|c|c|}
\hline \multirow{2}{*}{ LOTES } & \multirow{2}{*}{ Inicial } & \multicolumn{6}{|c|}{ EAT } & \multicolumn{6}{|c|}{ EASSS } \\
\hline & & $12 \mathrm{~h}$ & $24 \mathrm{~h}$ & $48 \mathrm{~h}$ & $72 \mathrm{~h}$ & $96 \mathrm{~h}$ & $120 \mathrm{~h}$ & $12 \mathrm{~h}$ & $24 \mathrm{~h}$ & $48 \mathrm{~h}$ & $72 \mathrm{~h}$ & $96 \mathrm{~h}$ & $120 \mathrm{~h}$ \\
\hline 1 & 11,1 & 12,4 & 14,0 & 26,2 & 27,1 & 28,9 & 33,1 & 12,4 & 12,5 & 16,0 & 17,8 & 29,2 & 29,2 \\
\hline 2 & 12,1 & 12,9 & 13,2 & 25,5 & 26,0 & 26,5 & 27,9 & 12,3 & 13,2 & 15,2 & 16,2 & 18,1 & 18,3 \\
\hline 3 & 12,1 & 13,7 & 14,0 & 26,0 & 26,8 & 27,6 & 28,3 & 12,6 & 13,1 & 15,1 & 16,4 & 19,0 & 19,1 \\
\hline 4 & 12,3 & 12,9 & 14,8 & 26,8 & 26,9 & 28,3 & 30,1 & 12,4 & 12,5 & 16,1 & 18,1 & 20,6 & 21,3 \\
\hline
\end{tabular}

Tabela 3. Germinação (\%) de quatro lotes de sementes de berinjela, cv. Napoli, após os períodos de envelhecimento acelerado tradicional (EAT), com solução salina saturada (EASSS) de quatro lotes de sementes de sorgo sacarino

\begin{tabular}{|c|c|c|c|c|c|c|c|c|c|c|c|c|}
\hline \multirow{2}{*}{ LOTES } & \multicolumn{6}{|c|}{ EAT } & \multicolumn{6}{|c|}{ EASSS } \\
\hline & $12 \mathrm{~h}$ & $24 \mathrm{~h}$ & $48 \mathrm{~h}$ & $72 \mathrm{~h}$ & $96 \mathrm{~h}$ & $120 \mathrm{~h}$ & $12 \mathrm{~h}$ & $24 \mathrm{~h}$ & $48 \mathrm{~h}$ & $72 \mathrm{~h}$ & $96 \mathrm{~h}$ & $120 \mathrm{~h}$ \\
\hline 1 & $86 a^{*}$ & $85 a$ & $70 \mathrm{c}$ & $66 b$ & $50 \mathrm{~b}$ & $28 b$ & $89 a$ & $75 b$ & $75 b$ & $75 b$ & $72 b$ & $67 b$ \\
\hline 2 & $88 \mathrm{a}$ & $87 a$ & $85 a$ & $79 a$ & $70 \mathrm{a}$ & $48 \mathrm{a}$ & $91 \mathrm{a}$ & $89 a$ & $87 a$ & $85 a$ & $85 a$ & $75 a$ \\
\hline 3 & $90 \mathrm{a}$ & $86 a$ & $77 b$ & $75 a$ & $71 \mathrm{a}$ & $42 \mathrm{a}$ & $88 a$ & $90 a$ & $83 a$ & $80 a$ & $85 a$ & $67 b$ \\
\hline 4 & $85 a$ & $81 \mathrm{a}$ & $54 d$ & $48 \mathrm{c}$ & $39 c$ & $28 \mathrm{~b}$ & $88 \mathrm{a}$ & $65 c$ & $61 c$ & $60 c$ & $58 \mathrm{c}$ & $53 \mathrm{c}$ \\
\hline $\mathrm{CV}(\%)$ & \multicolumn{6}{|c|}{5,7} & \multicolumn{6}{|c|}{3,7} \\
\hline
\end{tabular}

*Médias seguidas pela mesma letra minúsculas na coluna não diferem entre si pelo teste de Tukey a 5\% de probabilidade.

Tabela 4. Coeficiente de correlação de Pearson (r) entre os resultados dos testes de germinação (GER), primeira contagem da germinação (PC) e emergência em campo (ECP) com os testes de envelhecimento acelerado tradicional (EAT) e com solução salina saturada (EASSS), realizados em sementes de sorgo sacarino

\begin{tabular}{|c|c|c|c|c|}
\hline \multicolumn{2}{|c|}{ EA } & GER & PCG & EC \\
\hline \multirow{2}{*}{$12 \mathrm{~h}$} & EAT & $0,12^{\mathrm{ns}}$ & $0,22^{\mathrm{ns}}$ & $0,33^{\mathrm{ns}}$ \\
\hline & EASSS & $0,54^{* *}$ & $0,03^{\mathrm{ns}}$ & $0,52^{* *}$ \\
\hline \multirow{2}{*}{$24 \mathrm{~h}$} & EAT & $0,36^{\mathrm{ns}}$ & $0,17^{\mathrm{ns}}$ & $0,48^{\mathrm{ns}}$ \\
\hline & EASSS & $0,20^{\mathrm{ns}}$ & $0,35^{\mathrm{ns}}$ & $0,72^{*}$ \\
\hline \multirow{2}{*}{$48 \mathrm{~h}$} & EAT & $0,36^{\mathrm{ns}}$ & $0,42^{\mathrm{ns}}$ & $0,82^{*}$ \\
\hline & EASSS & $0,18^{\mathrm{ns}}$ & $0,37^{\mathrm{ns}}$ & $0,76^{*}$ \\
\hline \multirow{2}{*}{$72 \mathrm{~h}$} & $\overline{\text { EAT }}$ & $0,20^{\mathrm{ns}}$ & $0,43^{\text {ns }}$ & $0,70^{*}$ \\
\hline & EASSS & $0,25^{\mathrm{ns}}$ & $0,38^{\mathrm{ns}}$ & $0,78^{*}$ \\
\hline \multirow{2}{*}{$96 \mathrm{~h}$} & EAT & $0,21^{\mathrm{ns}}$ & $0,42^{\mathrm{ns}}$ & $0,73^{*}$ \\
\hline & EASSS & $0,21^{\mathrm{ns}}$ & $0,40^{\mathrm{ns}}$ & $0,69^{*}$ \\
\hline \multirow{2}{*}{$120 \mathrm{~h}$} & EAT & $0,27^{\mathrm{ns}}$ & $0,37^{\mathrm{ns}}$ & $0,71^{*}$ \\
\hline & EASSS & $0,38^{\mathrm{ns}}$ & $0.31^{\mathrm{ns}}$ & $0,82^{*}$ \\
\hline
\end{tabular}

${ }^{\text {ns }}=$ não significativo; $* *$ significativo a $5 \%$ de probabilidade; * significativo a $1 \%$ de probabilidade

\section{CONCLUSÕES}

A exposição das sementes por $24 \mathrm{~h}$ a $41{ }^{\circ} \mathrm{C}$ no envelhecimento acelerado com uso de solução saturada de $\mathrm{NaCl}$ e por $48 \mathrm{~h}$ a $41{ }^{\circ} \mathrm{C}$ no envelhecimento acelerado tradicional são eficientes na estratificação dos lotes de sementes de sorgo sacarino, possibilitando a classificação de lotes semelhante àquela que se obtêm no teste de emergência de plântulas.

\section{REFERÊNCIAS}

BERTOLIN, D. C.; SÁ, M. E. de; MOREIRA, E. R. Parâmetros do teste de envelhecimento acelerado para determinação do vigor de sementes de feijão. Revista Brasileira de Sementes, Londrina, v. 33, n. 1, p. 104 - 112, 2011.
BRASIL. Ministério da Agricultura, Pecuária e Abastecimento. Secretaria de Defesa Agropecuária. Regras para análise de sementes. Brasília: Mapa/ACS, 2009. 399p.

BRAZ, M. R. S.; ROSSETTO, C. A. V. Correlação entre testes para avaliação da qualidade de sementes de girassol e emergência das plântulas em campo. Ciência Rural, Santa Maria, v.39, p. 2004-2009, 2009.

COIMBRA, R. A., MARTINS, C. C.; TOMAZ, C. A.; NAKAGAWA, J. Testes de vigor utilizados na avaliação da qualidade fisiológica de sementes de milho-doce (sh2). Ciência Rural, Santa Maria, v.39, p.2402-2408, 2009.

DUTRA, A. S.; VIEIRA, R. D. Envelhecimento acelerado como teste de vigor para sementes de milho e soja. Ciência Rural, Santa Maria, v.34, n.3, p.715-721, 2004. 
DURÃES, F. O. M. Agroenergia em Revista, Ed. 3, p.7, 2011. Disponível em: http://ainfo.cnptia.embrapa.br/digital/bitstream/item/56043/1/ BolfeAgroeRevis.pdf

GUISCEM, J. M.; ZUCARELI, C.; NAKAGAWA, J.; ZANOTTO, M. D. Correlação de testes de vigor com emergência no campo e germinação em laboratório em sementes de milho doce BR 400 (gene bt). Informativo ABRATES, v.11, n.2, p.107, 2001.

IBRAHIM, A. E.; TEKRONY, D. M.; EGLI, D. B. Accelerated aging techniques for evaluating sorghum seed vigor. Journal of Seed Technology, v.17, n.1, p.29-37, 1993.

JAIN, N.; KOOPAR, R.; SAXENA, S. Effect of accelerated ageing on seeds of radish (Raphanus sativus L.). Asian Journal of Plant Sciences, Ásia, v.5, n.3, p.461-464, 2006.

JIANHUA, Z.; McDONALD, M. D. The saturated salt accelerated aging test for small-seeded crops. Seed Science and Technology, v. 25, n.1, p.123-131, 1996.

LEMES, E. S.; OLIVEIRA, S. de; ALMEIDA, A. da S.; MENEGHELLO, G. E.; GEWEHR, E.; TUNES, L. M. de. Testes de vigor para avaliação da qualidade de sementes de grama-bermuda. Revista de la Facultad de Agronomía, v.114, n.2, p. 185-192, 2015.

LOPES, R. R.; FRANKE, L. B.; NUNES F. S. Metodologia alternativa do teste de envelhecimento acelerado para sementes de azevém. Scientia Agrária, v.10, n.2. p.80-94, 2009.

MACHADO, A.; CONCEIÇÃO, A. R. Programa estatístico WinStat: sistema de análise estatístico para Windows. Pelotas, RS. 2003.

MARCOS FILHO, J. Teste de envelhecimento acelerado. In: KRZYZANOWSKI, F. C.; VIEIRA, R. D.; FRANÇA NETO, J. B. (Eds.). Vigor de sementes: conceitos e testes. Londrina: Abrates, 1999. p.1-24.

MARCOS FILHO, J. Teste de envelhecimento acelerado. In: VIEIRA, R. D.; CARVALHO, N. M. Testes de vigor em sementes. Jaboticabal: FUNEP, 1994. p.133-149.

MARCOS FILHO, J.; NOVEMBRE, A. D. L. C. Avaliação do potencial fisiológico de sementes de hortaliças. In: NASCIMENTO, W. M. (Ed.). Tecnologia de sementes de hortaliças. Brasília, DF: Embrapa Hortaliças, 2009. p.185246.

MENEZES, N. L. Testes Rápidos. Seed News, v. 5, n. 3, p. 30-32, maio/jun. 2001

MIRANDA, D. M. de; NOVEMBRE, A. D. da L. C.; CHAMMA, H. M. C. P. Avaliação do potencial fisiológico de sementes de sorgo pelo teste de envelhecimento acelerado. Revista Brasileira de Sementes, Pelotas, v. 23, n.1, p.226-231, 2001.

MOREIRA, L. R.; MIRANDA, G. V.; ANJOS, R. S. R. dos; ERVILHA, J. D. C.; SILVA, J. C. V.; COUTINHO, P. H.; JÚNIOR, G. A. de C. Avaliação de características fisiológicas e morfológicas de sorgo sacarino em resposta ao déficit hídrico. XXVIII Congresso Nacional de Milho e Sorgo, p.132-138, 2010.
NAKAGAWA, J. Testes de vigor baseados no desempenho das plântulas. In: KRZYZANOWSKI, F. C.; VIEIRA, R. D.; FRANÇA NETO, J. B. (eds). Vigor de sementes: conceitos e testes. p. 2-21. 1999.

NERY, M. C.; CARVALHO, M. L. M.; GUIMARÃES, R. M. Testes de vigor para avaliação da qualidade de sementes de nabo forrageiro. Informativo ABRATES, v.19, p. 09-20, 2009.

PARRELlA, N. N. L. D.; PARRELlA, R. A. da C.; RIBEIRO, A. M. P.; SILVA, S. R. da. Comportamento de genótipos de sorgo sacarino após armazenamento. XXIX Congresso Nacional de Milho e Sorgo, p. 3565-3569, 2012.

PARRElla, N. N. L. D.; PARRElla, R. A. da C. Agroenergia em Revista, Ed. 3, p.14, 2011.

PEDROSO, D. C.; TUNES, L. M. de; BARBIERI, A. P.; BARROS, A. C. S. A.; MUNIZ, M. F. B.; MENEZES, V. O. Envelhecimento acelerado em sementes de trigo. Ciência Rural, Santa Maria, v. 40, n. 11, p. 2389-2392, 2010.

SANTOS, F. G.; CASELA, C. R.; WAQUIL, J. M. Melhoramento de espécies cultivadas. Viçosa: Ed. UFV, p. 605-658. 2005.

SOUZA, C. C.; DANTAS, J. P.; SILVA, S. M.; SOUZA, V. C.; ALMEIDA, F. A.; SILVA, L. E. Produtividade do sorgo granífero cv. Sacarino e qualidade de produtos formulados isoladamente ou combinados ao caldo de cana-de-açúcar. Ciência e Tecnologia de Alimentos, Campinas, v. 25, n.3, p. $512-517,2005$.

SOUZA, F. H. D.; MARCOS-FILHO, J. Estudo comparativo de métodos para a avaliação do vigor de sementes de sorgo (Sorghum vulgare Pers). Anais da Escola Superior de Agricultura "Luiz de Queiroz", São Paulo, v.32, p.369-383, 1975.

TORRES, S. B.; MARCOS FILHO, J. Teste de envelhecimento acelerado em sementes de maxixe (Cucumis anguria L.). Revista Brasileira de Sementes, Pelotas, v.23, n.2, p.108-112, 2001.

TUNES, L. M.; PEDROSO, D. C.; BADINELLI, P. G.; TAVARES, L. C.; RUFINO, C. A.; BARROS, A. C. S. A.; MUNIZ, M. F. B. Envelhecimento acelerado em sementes de cebola com e sem solução salina saturada. Ciência Rural, Santa Maria, v. 41, n. 1, p. 33-37, 2011.

TUNES, L. M. de; TAVARES, L. C.; BARROS, A. C. S. A. Envelhecimento acelerado como teste de vigor para sementes de arroz. Revista de Ciências Agrárias, Lisboa, v.35, n.1, p. 120-127, 2012.

VAZQUEZ, G. H.; BERTOLIN, D. C.; SPEGIORIN, C. N. Testes de envelhecimento acelerado e de condutividade elétrica para avaliar a qualidade fisiológica de sementes de sorgo (Sorghum bicolor (L.) Moench). Revista Brasileira de Biociências, Porto Alegre, v. 9, n. 1, p. 18-24, 2011.

VENANCIO, L.P.; LOPES, J.P.; MACIEL, K.S.; COLA, M.P.A. Teste do envelhecimento acelerado para avaliação da qualidade fisiológica de sementes de milho. ENCICLOPÉDIA BIOSFERA, Centro Científico Conhecer Goiânia, v.8, N.14; p.899 - 906, 2012. 